\title{
Label-Free Imaging of Cerebral $\beta$-Amyloidosis with Extended-Focus Optical Coherence Microscopy
}

\author{
Tristan Bolmont, ${ }^{1,2 \star}$ Arno Bouwens, ${ }^{2 \star}$ Christophe Pache, ${ }^{2}$ Mitko Dimitrov, ${ }^{1}$ Corinne Berclaz, ${ }^{2}$ Martin Villiger, ${ }^{2}$ \\ Bettina M. Wegenast-Braun, ${ }^{3,4}$ Theo Lasser, ${ }^{2 \star}$ and Patrick C. Fraering ${ }^{1 \star}$ \\ ${ }^{1}$ Laboratory of Molecular and Cellular Biology of Alzheimer's Disease, Brain Mind Institute and School of Life Sciences, Ecole Polytechnique Fédérale de \\ Lausanne (EPFL), CH1015 Lausanne, Switzerland; '2Laboratoire d'optique Biomédicale, Institute of Microengineering, School of Engineering, EPFL, \\ CH1015 Lausanne, Switzerland; ${ }^{3}$ Department of Cellular Neurology, Hertie Institute for Clinical Brain Research, University of Tübingen, DE72076 \\ Tübingen, Germany; and ${ }^{4}$ DZNE-German Center for Neurodegenerative Diseases, DE72076 Tübingen, Germany
}

We demonstrate label-free imaging of cerebral $\beta$-amyloidosis ex vivo and in a living mouse model of Alzheimer's disease using extendedfocus Fourier domain optical coherence microscopy (xfOCM). xfOCM provides 3D, high-resolution images of individual $\beta$-amyloid plaques in the brain parenchyma and vasculature and requires no staining of the Alzheimeric sample under investigation. xfOCM also opens the possibility to perform minimally invasive studies of $\beta$-amyloid pathology in vivo, without the use of labeling methods, which potentially confound experimental findings.

\section{Introduction}

Alzheimer's disease (AD) is pathologically characterized by the aggregation of misfolded proteins accumulating as fibrillar amyloid deposits in vulnerable regions of the CNS. The amyloid cascade hypothesis proposes that the oligomerization and fibrillization of amyloid- $\beta(\mathrm{A} \beta)$ in the brain is an early and critical event that triggers a cascade of pathological events leading to hyperphosphorylation and somatodendritic segregation of tau, formation of neurofibrillary lesions, neuroinflammation, neurodegeneration, and, finally, dementia (Hardy and Selkoe, 2002). Thus, the visualization of cerebral $\mathrm{A} \beta$ amyloidosis is of critical importance for the evaluation of its progression and the development of new $\mathrm{A} \beta$-based therapeutics. The natural history of $\mathrm{A} \beta$ plaques has been extensively studied in Alzheimeric mice with multiphoton microscopy techniques using amyloid-binding dyes such as thioflavin or Congo red derivatives (Christie et al., 2001; Bacskai and Hyman, 2002; Klunk et al., 2002). Nevertheless, these

Received Feb. 27, 2012; revised Aug. 16, 2012; accepted Aug. 22, 2012.

Author contributions: T.B., A.B., T.L., and P.C.F. designed research; T.B., A.B., C.P., M.D., and M.V. performed research; C.B. and B.M.W.-B. contributed unpublished reagents/analytic tools; T.B. and A.B. analyzed data; T.B., A.B., T.L., and P.C.F. wrote the paper.

This work was supported by the Swiss National Science Foundation Grant 310000-116652/1 (P.C.F.), the Strauss Foundation (T.B. and P.C.F.), and partly by the SystemsX project Grant KK201-542 (A.B.) and a European Union Grant EU FP7-222980 (M.V.). We thank Professor M. Jucker (Hertie Institute of Clinical Brain Research, University of Tübingen) for providing the APPPS1 and the APP Dutch brains as well as for insightful comments on this manuscript. We thank Dr. M. Staufenbiel (Novartis, Basel) for the APP23 brains. We are also grateful to Professor D. Walsh (Harvard Institutes of Medicine, Boston) for the generous gift of DW6 antibody, and to Professor W. Klunk for providing generous amounts of the amyloid-binding dye methoxy-X04.

*T.B., A.B., T.L., and P.C.F. contributed equally to this work.

The authors declare no competing financial interests.

Correspondence should be addressed to either of the following: Professor Patrick C. Fraering, Brain Mind Institute and School of Life Sciences, Ecole Polytechnique Fédérale de Lausanne, CH1015 Lausanne, Switzerland, E-mail: patrick.fraering@epfl.ch; or Professor Theo Lasser, Laboratoire d'optique Biomédicale, Institute of Microengineering, School of Engineering, Ecole Polytechnique Fédérale de Lausanne, CH1015 Lausanne, Switzerland, E-mail: theo.lasser@epfl.ch.

DOI:10.1523/JNEUROSCI.0925-12.2012

Copyright $\odot 2012$ the authors $\quad 0270-6474 / 12 / 3214548-09 \$ 15.00 / 0$ amyloid-binding compounds have been reported to alter the formation of amyloid structures (Lee, 2002; Cohen et al., 2009), and thus their use in longitudinal in vivo imaging studies may confound experimental findings. To avoid potential interference, we introduce a method derived from optical coherence tomography (OCT) for label-free imaging of cerebral A $\beta$ amyloidosis ex vivo and in the brain of a living transgenic mouse model of AD (Radde et al., 2006). OCT is an interferometric technique that provides 3D images with micrometric resolution from within biological tissues (Izatt et al., 1994; Fercher et al., 2003; Fujimoto, 2003). Image contrast in OCT is provided by the intrinsic light-scattering properties of the sample under investigation. The axial resolution is determined solely by the spectral width of the light. Therefore, unlike confocal microscopy, it is independent of the microscope objective or pinhole size. However, the lateral resolution is given by the numerical aperture (NA) of the objective, thereby creating a trade-off between transverse resolution and focal range. To circumvent this trade-off, we have developed an extended-focus version of OCT (extended-focus Fourier domain optical coherence microscopy; xfOCM) that exhibits uniformly high transverse resolution along an extended focal range (Leitgeb et al., 2006; Villiger et al., 2009). To this end, a Bessel-mode instead of the traditional Gaussian mode illumination beam is used (Leitgeb et al., 2006; Villiger et al., 2009). Additionally, Bessel beams exhibit a self-reconstructing capability, making them particularly interesting for deep imaging into scattering tissues (Fahrbach et al., 2010). The prototype xfOCM instrument employs nearinfrared linearly polarized light from a broad-bandwidth laser source $\left(\lambda_{0}=800 \mathrm{~nm}, \Delta \lambda=135 \mathrm{~nm}\right)$. The detection in the Fourier domain endows xfOCM with high sensitivity and fast acquisition (Leitgeb et al., 2003). To demonstrate ex vivo xfOCM imaging of parenchymal and vascular $\mathrm{A} \beta$ plaques, we have imaged unfixed and unlabeled brains from three different transgenic models of cerebral $\mathrm{A} \beta$ amyloidosis. Without administration of contrast 
agents, we then followed cerebral $\mathrm{A} \beta$ plaque pathology in the living APPPS1 transgenic mouse through xfOCM. To further support the conclusion that structures imaged by $\mathrm{xfOCM}$ are indeed $\mathrm{A} \beta$ plaques, the $\mathrm{xfOCM}$ instrument was complemented with a confocal fluorescence channel, allowing simultaneous observation and comparison of the same amyloid-containing brain areas by the two imaging modalities.

\section{Materials and Methods}

Mice. For the in vivo imaging studies, APPPS1 transgenic mice [obtained from Professor M. Jucker, Hertie Institute of Clinical Brain Research (HIH), University of Tübingen, Germany] were maintained at the Ecole Polytechnique Fédérale de Lausanne animal core facility. APPPS1 animals coexpress the KM670/671NL Swedish mutation of human amyloid precursor protein (APP) and the L166P mutation of human presenilin 1 (PS1) under the control of the Thy-1 promoter, and show age-dependent accumulation of parenchymal $\mathrm{A} \beta$ plaques with minimal vascular $\mathrm{A} \beta$ amyloid that is restricted to the pial vessels (Radde et al., 2006). APPPS1 mice were generated on a C57BL/6 background. Both male and female APPPS1 mice as well as aged-matched control nontransgenic littermates were used. Mice were housed in groups of five in pathogen-free conditions until surgical intervention, after which they were singly housed. For ex vivo imaging, in addition to APPPS1 brains, we used brains from APP23 mice (obtained from Dr. M. Staufenbiel, Novartis, Basel) and from APP Dutch mice (obtained from Professor M. Jucker, HIH, University of Tübingen, Germany). APP23 mice overexpress human APP with the KM670/671NL Swedish mutation and show first $A \beta$ deposits in the neocortex at $\sim 6$ months of age (Sturchler-Pierrati et al., 1997). As mice age, plaques increase in both size and number and eventually occupy a substantial area of the neocortex. In 24-month-old APP23 mice, prominent deposition of cerebrovascular amyloid is observed. APP Dutch mice overexpress human E693Q APP in neurons, leading to extensive cerebral amyloid angiopathy (CAA), smooth muscle cell degeneration, hemorrhages, and neuroinflammation. This mouse model develops robust CAA in the absence of parenchymal amyloid (Herzig et al., 2004).

$x f O C M$ instrument and data recording. The layout of the XfOCM instrument used for this study was based on a Mach-Zehnder interferometer with separate illumination and detection paths, as shown in Figure 1. A femtosecond laser (Femtosecond Compact Pro; Femtolasers) emitting broad-bandwidth, linearly polarized light $\left(\lambda_{0}=800 \mathrm{~nm}, \Delta \lambda=135 \mathrm{~nm}\right)$ was used as a low temporal coherence light source. A first beam splitter separated the laser beam into the reference and sample arms of the imaging interferometer. The sample arm contained a conical lens (axicon) to create a Bessel-like illumination beam, which was raster scanned in the lateral dimensions by two galvanometric scanners. A $10 \times$ Zeiss Neofluar objective (NA 0.3, Carl Zeiss) guided the sample beam into the brain, where a Bessel-like illumination pattern was formed in the axial direction. The resulting illumination beam propagated nearly diffraction-free over a distance of $400 \mu \mathrm{m}$ into the brain, with the central lobe maintaining a uniform lateral definition of $1.3 \mu \mathrm{m}$. In the axial direction (i.e., along the optical axis), the resolution depends on the central wavelength and the spectral width of the light source, which resulted in an axial resolution of $2 \mu \mathrm{m}$ in biological tissues. The illumination power on the brain was $5 \mathrm{~mW}$. Image contrast in XfOCM is provided by the intrinsic light-scattering properties of the brain sample under investigation. Part of the illumination is elastically scattered by the brain structure into the backward direction and collected by the same objective. This captured light was descanned by means of the scanning system. A second beam splitter had the dual function of separating the illumination and detection paths and combining the detection light with the reference beam. A custom spectrometer based on a transmission grating (1200 lines/mm), an objective lens $(\mathrm{f}=135 \mathrm{~mm}$ ), and a line detector with 2048 pixels (Atmel Aviiva M2) recorded the resulting interference pattern with an acquisition rate of $20 \mathrm{kHz}$. The detection in the Fourier domain endows xfOCM with high sensitivity and fast acquisition. The data acquisition time for typical sample volumes in the present work was $\sim 5 \mathrm{~s}$. Finally, the brain structure was reconstructed using the following signal processing on the recorded spectra. First, an averaged background signal was subtracted from the spectra, followed by a wavelength to wave number mapping. In a last step, the mapped spectra were Fourier transformed and the squared norm was scaled logarithmically to provide depth profiles of the brain structure. The image stacks were rescaled along the axial direction using an estimated index of refraction of $n=1.33$ to represent geometrical distance instead of optical distance. We used three sampling protocols: (1) a fast sampling protocol with 512 samples for $800 \mu \mathrm{m}$ laterally and 300 depth slices over $600 \mu \mathrm{m},(2)$ a second fast sampling protocol with 512 samples for $1000 \mu \mathrm{m}$ laterally and 300 depth slices over $600 \mu \mathrm{m}$, and (3) a slower and denser sampling protocol with 1024 samples for 800 $\mu \mathrm{m}$ laterally and 300 depth slices over $600 \mu \mathrm{m}$. Protocols (1) and (2) were used for fast, high-resolution in vivo and ex vivo imaging experiments; protocol (3) was used for maximum-resolution ex vivo imaging.

Fluorescence imaging coupled to the XfOCM instrument for comparison of amyloid morphologies between the two modalities. A confocal fluorescence imaging channel was added collinearly with the xfOCM beam path (Fig. 1). The fluorescence and xfOCM imaging modalities shared the beam-scanning system and microscope objective $(10 \times$ Zeiss Neofluar, NA 0.3). In this way, xfOCM and fluorescence images were recorded simultaneously. The confocal channel contains a fluorescence filter set and an adjustable pinhole. Fluorescence excitation and emission light were separated from the xfOCM signal using a dichroic mirror (longpass, cutoff at $720 \mathrm{~nm}$ ). Excitation light was provided by a supercontinuum laser (Koheras SuperK Extreme; NKT Photonics), which was filtered to remove the infrared part of the spectrum (dichroic mirror, cutoff at 724 $\mathrm{nm}$ and KG1 infrared absorber) before passing through the excitation filter (bandpass, 450-490 nm, Zeiss filter set 10). Excitation and emission light were split by a dichroic mirror (longpass, cutoff at $510 \mathrm{~nm}$, Zeiss filter set 10). Detection was done through an emission filter (bandpass, 515-565 nm, Zeiss filter set 10) and an avalanche photodiode (SPCM-AQR-14-FC; PerkinElmer). Control experiments confirmed the separation of fluorescence and XfOCM signals. The pinhole size was optimized for optimal image contrast. Under these settings, a confocal image corresponded to a depth projection of $\sim 60 \mu \mathrm{m}$ in the corresponding xfOCM image stack. A systemic injection of an amyloid-binding compound was chosen to permit its homogenous distribution in relatively thick tissue sections ex vivo $(1000 \mu \mathrm{m})$. To this end, APPPS1 mice $(n=2)$ were injected intraperitoneally with $10 \mathrm{mg} / \mathrm{kg}$ of the Congo red derivate methoxy-X04 (Klunk et al., 2002) $(5 \mathrm{mg} / \mathrm{ml}$ in 50\% dimethylsulfoxide; $50 \% \mathrm{NaCl}, \mathrm{pH} 12$ ), as previously described (Bolmont et al., 2008). After $24 \mathrm{~h}$, the injected mice were deeply anesthetized and the brains were removed, snap-frozen, and stored at $-80^{\circ} \mathrm{C}$ until sectioning in a cryostat to collect $1000 \mu \mathrm{m}$ sagittal sections. Subsequently and without additional manipulation, sections encompassing the neocortex and hippocampus were de-frozen and directly observed simultaneously by fluorescence microscopy and xfOCM.

Experimental design for ex vivo $x f O C M$ imaging. Unfixed samples were obtained from snap-frozen mice brains. To demonstrate the feasibility of imaging $\mathrm{A} \beta$ plaque pathology ex vivo by xfOCM, a total of five unfixed brains from adult, 10 - to 12 -month-old, $\mathrm{A} \beta$-depositing APPPS1 mice as well as five control brains from aged-matched nontransgenic mice and three brains from young, 1-month-old, predepositing APPPS1 mice were used. Regions corresponding to the parietal cortex were imaged, and $x y z$ stacks corresponding to $400^{2}, 800^{2}$, or $1000^{2} \times 600 \mu \mathrm{m}^{3}$ were reconstructed. Additionally, we acquired $x y z$ stacks (corresponding to $800^{2} \times 600 \mu \mathrm{m}^{3}$ ) from coronal brain slices (2000 $\mu \mathrm{m}$ thickness) encompassing cortical mouse brain regions. An average of $25 x y z$ locations was imaged for each brain ex vivo. To demonstrate the ability of xfOCM to image cerebrovascular $\mathrm{A} \beta$ deposits, we used unfixed brains from aged ( 24 months old, $n=3$ ), adult ( 12 months old, $n=3$ ), and young ( 4 months old, $n=3$ ) APP23 mice. We also used unfixed brains from aged APP Dutch transgenic mice (27 to 28 months old, $n=3$ ) and aged-matched nontransgenic mice $(n=2)$. For APP23 and APP Dutch brains, we acquired $x y z$ stacks corresponding to $800^{2} \times 600 \mu \mathrm{m}^{3}$ from cortical brain regions (25xyz locations imaged for each brain). The ex vivo transversal study was performed on unfixed brains obtained from APPPS1 mice of different age groups: predepositing ( 1 month old), young ( 2 and 4 months old), adult (8 months old), and aged (18 months old) ( $n=3$ brains per group). We acquired $x y z$ stacks corresponding to $800^{2} \times 600 \mu \mathrm{m}^{3}$ from 


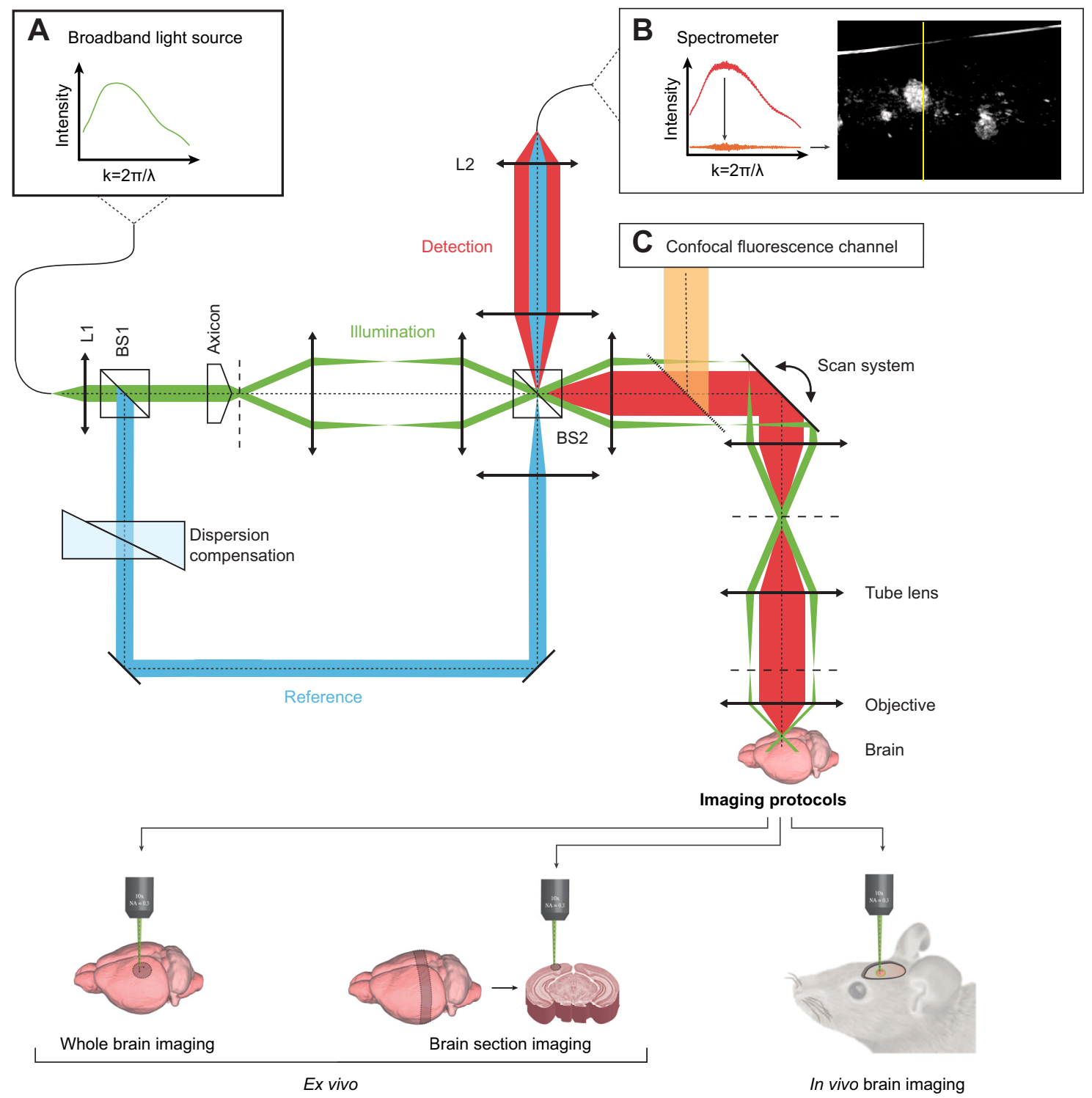

Figure 1. Schematic illustration of the xfOCM instrument with decoupled illumination and detection paths. Light of a laser source with a broad spectrum ( $A$, inset) is collimated by lens $\mathrm{L} 1$ and separated by beam-splitter BS1 into sample (green beam) and reference arms (blue beam) of the imaging interferometer. The axicon in the sample arm generates a Bessel-like illumination beam, which is subsequently raster scanned in the lateral directions. The objective images the illumination beam into the sample and collects backscattered light. Beam-splitter BS2 combines the reference beam with the backscattered light. Finally, lens L2 focuses the light into an optical fiber to guide it to the spectrometer. The recorded interference pattern is processed to yield a depth profile of the sample structure ( $\boldsymbol{B}$, inset). A confocal fluorescence channel is superimposed on the xfOCM channel by means of a dichroic mirror ( $\boldsymbol{C}$, inset).

regions corresponding to the parietal cortex (five $x y z$ locations imaged for each brain).

Experimental design for in vivo xfOCM imaging. A total of five $\mathrm{A} \beta$ depositing adult APPPS1 mice (10 to 12 months old) were used to demonstrate the feasibility of imaging $\mathrm{A} \beta$ plaque pathology by xfOCM in the living mouse brain, as well as to determine the distribution and size of $\mathrm{A} \beta$ deposits detected in vivo. In a parallel set of experiments, three young predepositing APPPS1 mice (1 month old) and five adult nontransgenic mice were used as controls. Data collected from imaging the parietal cortex of the mouse allowed the reconstruction of three types of $x y z$ stacks, with $x y$ surfaces corresponding to $400^{2}, 800^{2}$, or $1000^{2} \mu \mathrm{m}^{2}$, over a depth of $z=600 \mu \mathrm{m}$ below the cortical surface. An average of $15 x y z$ locations was imaged for each animal. For chronic imaging, one protocol was used to study short-term amyloid dynamics: $800^{2} \times 600 \mu \mathrm{m}^{3} x y z$ stacks every $30 \mathrm{~s}, 8$ sessions; $n=5$ adult APPPS1; $n=3$ adult nontransgenic mice. Moreover, two distinct long-term imaging protocols were used: (1) $800^{2} \times 600 \mu \mathrm{m}^{3} x y z$ stacks every $24 \mathrm{~h}, 2$ sessions; $n=2$ adult APPPS $1 ; n=2$ adult nontransgenic and (2) $800^{2} \times 600 \mu \mathrm{m}^{3} x y z$ stacks every $7 \mathrm{~d}, 5$ sessions; $n=4$ young ( 3 months old) APPPS1. An average of five $x y z$ locations was imaged across all sessions for each mouse. During the initial imaging session, the imaged location was selected in a semirandom manner, with the only exclusion criteria being the presence of a large vessel on or below the cortical surface. During the subsequent imaging sessions, the image spot was first repositioned manually using a live image taken through the microscope objective under white light illumination of large blood vessels under the glass window. Precise registration of the XfOCM image stacks was done in a postprocessing step based on the 3D structure of the brain's vasculature. The 3D structure of blood vessels was obtained by label-free optical micro-angiography of the same brain location. Micro-angiography images were acquired using a previously reported scan protocol (Srinivasan et al., 2010) implemented on the xfOCM instrument. The micro-angiography data stacks were processed using a phase variance algorithm (Vakoc et al., 2009). Phase variance images reveal only moving light scatterers (e.g., red blood cells), thereby enabling the xfOCM instrument with a second contrast method. The translation and rotation parameters for registration were calculated from the micro-angiography image stacks and were subsequently applied to register the structural xfOCM stacks. 
Neurosurgery and in vivo imaging. All animal procedures were performed according to the guidelines of the local authorities and Swiss animal protection law. Mice were anesthetized, placed on a warming pad, and fixed in a Kopf stereotactic frame. A $2 \mathrm{~cm}$ long longitudinal cut was performed with a scalpel in the middle of the skull after the coat had been shaved and the skin disinfected with 70\% ethanol. The skin was folded and fixed on the side by four bulldog clamps. Lines forming a rectangle (from the midline -1 and $-4 \mathrm{~mm}$ and at -0.5 and $-3.5 \mathrm{~mm}$ from bregma) were gently drilled onto the skull surface. Using a pair of angled forceps, the circumscribed portion of the skull was then removed, being careful not to disturb or puncture the dura. To image $\mathrm{A} \beta$ plaque pathology, anesthetized animals were secured into a modified stereotactic frame and placed below the objective of the xfOCM instrument directly after craniotomy. Using custom real-time image previewing software, the distance between the mouse brain and the objective was precisely adjusted to bring the most superficial layers of the cortex within the instrument's imaging range. During imaging, artificial CSF was suffused over the surface of the brain. For long-term imaging, after realization of the craniotomy, a matching, custom-cut glass coverslip (130-170 $\mu \mathrm{m}$ thickness) was held with a micromanipulator and slowly lowered until it was parallel to the edge of the skull. Once correctly positioned, the coverslip was sealed to the skull with lightcuring dental cement (Flowline; Heraeus Kulzer). Animals were imaged 1 week after the surgical implantation.

Histology. Immediately after imaging, mice were deeply anesthetized and perfused transcardially with ice-cold PBS, $\mathrm{pH} 7.4$, followed by $4 \%$ paraformaldehyde (PFA) in ice-cold PBS. Brains were removed and postfixed overnight in the same fixative followed by $48 \mathrm{~h}$ incubation in $30 \%$ sucrose. Brains were frozen in 2-propanol (Merck) and subsequently sectioned on a freezing-sliding microtome to collect $25 \mu \mathrm{m}$ horizontal sections. Sections were immunostained to visualize $\mathrm{A} \beta$ deposits using rabbit polyclonal antibody (DW6, 1:500; kindly provided by Professor D. Walsh). Vectastain Elite ABC Kits were used and revelation obtained with Vector SG Blue (Vector Laboratories). For histological staining, brain sections were stained with cresyl violet according to standard protocols. To compare $\mathrm{A} \beta$ plaques imaged by xfOCM ex vivo with that in postmortem serial sections obtained from the same imaged areas, directly after imaging, brains from APPPS1 mice were fixed in 4\% PFA for $48 \mathrm{~h}$, followed by $48 \mathrm{~h}$ of incubation in $30 \%$ sucrose. Brains were frozen in 2-propanol, serial sectioned, and immunostained with anti-A $\beta$ antibody DW6.

Data analysis. The analysis of the first set of ex vivo and in vivo xfOCM experiments was done manually using the public domain National Institutes of Health ImageJ. Plaque numbers, distribution, and sizes were determined from $800^{2} \times 600 \mu \mathrm{m}^{3} x y z$ stacks $(n=12)$. The location of each plaque center was recorded manually in three dimensions, and the $z$ coordinates were used for calculation of $\mathrm{A} \beta$ plaque distribution in the $600 \mu \mathrm{m}$ of depth imaged. Plaques located on the edges of the stacks were excluded from analysis. Data reported are mean \pm SEM. The radii of A $\beta$ plaques were obtained from measuring half of the measured maximum straight distance that passes through the center of all plaques located between 100 and $300 \mu \mathrm{m}$ below the cortical surface. To process the large amount of data $\left(75\right.$ stacks of $\left.800^{2} \times 600 \mu \mathrm{m}^{3} x y z\right)$ from the ex vivo transversal xfOCM study, we implemented an automatic segmentation algorithm in the MATLAB programming environment to identify and quantify amyloid plaques in brain samples. The algorithm uses a Hidden Markov Random Field model for the intensity of the image pixels, similar to a previously published protocol (Zhang et al., 2001). Brains of three animals per age group were analyzed with five imaging spots $\left(800^{2} \times 600\right.$ $\mu \mathrm{m}^{3} x y z$ stacks) acquired per brain sample. Image stacks were preprocessed to remove background and optimize contrast. The statistics of pixels corresponding to amyloid plaque or other brain tissue were iteratively estimated and used to classify the pixels. The resulting 3D segmentations were validated manually. The subsequent quantification included the calculation of amyloid plaque load and average plaque volume per age group. The number and radii of all plaques were determined in the group of young, 2-month-old brain samples. Many of the figures and qualitative visualizations were postprocessed (Gaussian filter and background subtraction) with the assistance of Imaris software (Bitplane).
Quantification of the similarity between fluorescence and xfOCM imaging of plaques was done between the fluorescence images and their corresponding depth projections in xfOCM stacks. An average of five sections with five $x y$ locations per section were imaged, yielding a total of 25 locations imaged per animal, with $800^{2} \mu \mathrm{m}^{2} x y$ images that were recorded. The images were first segmented to allow unambiguous assessment of the spatial overlap of structures measured by both modalities. On the ensemble of segmented images, a colocalization coefficient was calculated, analogous to Manders' overlap coefficient (Manders et al., 1993). The colocalization coefficient of xfOCM to fluorescence imaging is given by the ratio of the plaque area jointly identified by both modalities to the total plaque area in the xfOCM image.

\section{Results}

We have demonstrated the feasibility of imaging $\mathrm{A} \beta$ plaque pathology by xfOCM in a first series of ex vivo experiments using unfixed brains from adult, 10- to 12-month-old, $\mathrm{A} \beta$-depositing APPPS1 transgenic mice, as well as from control, aged-matched, nontransgenic mice and young, 1 -month-old, pre-A $\beta$-depositing APPPS1 mice. The APPPS1 transgenic mouse line develops early and age-dependent accumulation of nearly $100 \%$ congophilic A $\beta$ plaques in the brain parenchyma with minimal vascular $\mathrm{A} \beta$ pathology that is restricted to the pial vessels (Radde et al., 2006). Imaging was first accomplished on the whole amyloid-laden brain and nontransgenic brain (Fig. $2 A, B$, respectively). $x y z$ datasets with $x y$ corresponding to $400^{2} \mu \mathrm{m}^{2}, 800^{2} \mu \mathrm{m}^{2}$ or $1000^{2} \mu \mathrm{m}^{2}$, over a depth of $600 \mu \mathrm{m}$, were recorded. The acquisition time for each dataset was $\sim 5 \mathrm{~s}$. Analysis of $x y z$ stacks obtained from the $\mathrm{A} \beta$-laden parietal cortex revealed that $\mathrm{A} \beta$ deposits of various sizes could be reconstructed up to $600 \mu \mathrm{m}$ below the surface of the brain (Figs. $2 A$, $3 A, D$ ), without prior $\mathrm{A} \beta$ staining or tissue processing. The origin of the image contrast between $A \beta$ plaques and the surrounding tissue components in xfOCM is based on their different light-scattering coefficients. We observed that $\mathrm{A} \beta$ plaques are revealed with a positive contrast, which implies a stronger light scattering, most probably attributed to both their structural organization (fibrils) and higher density. While lateral resolution is conserved over the extended focus, the depth penetration of xfOCM imaging is limited to $\sim 600 \mu \mathrm{m}$ due to the weaker light-scattering signal measured from plaques located below this depth (Fig. 3D). No amyloid structures comparable to those reconstructed in APPPS1 mice could be observed in the parietal cortex of nontransgenic mice (Fig. $2 B$ ). No difference in imaging was observed for brains from predepositing APPPS1 mice as compared with nontransgenic brains (data not shown), revealing that the structures detected by xfOCM in depositing mice were insoluble fibrous $\mathrm{A} \beta$ aggregates. In APPPS 1 mice, imaging of $\mathrm{A} \beta$ plaques was also achieved on coronal brain slices ( 2 $\mathrm{mm}$ thickness) encompassing the cortex (Fig. 2C). Notably, large bundles of neural fibers such as the corpus callosum were revealed with a positive contrast (Fig. $2 C$, asterisk). To confirm that observed structures in amyloid-laden brains are indeed $\mathrm{A} \beta$ plaques, brains of APPPS1 mice were fixed immediately after imaging, serial sectioned, and immunostained with an antibody against A $\beta$ (DW6, 1:500). Subsequent microscopic images of A $\beta$-stained APPPS1 brain sections confirmed the amyloid morphologies imaged ex vivo by xfOCM (Fig. 2D). In a second series of ex vivo experiments, we demonstrated xfOCM imaging of cerebrovascular $\mathrm{A} \beta$ pathology, using unfixed brains from aged A $\beta$-depositing APP23 and APP Dutch transgenic mice (Sturchler-Pierrat et al., 1997; Herzig et al., 2004). Whereas APPPS1 mice have virtually no $A \beta$ deposition in the vasculature (Radde et al., 2006), APP23 mice develop cerebrovascular amyloid in addition to parenchymal plaques (Calhoun et al., 1999), and APP Dutch mice show $\mathrm{A} \beta$ deposition exclusively in the brain vasculature (Herzig et al., 2004). Analysis of $x y z$ stacks ob- 

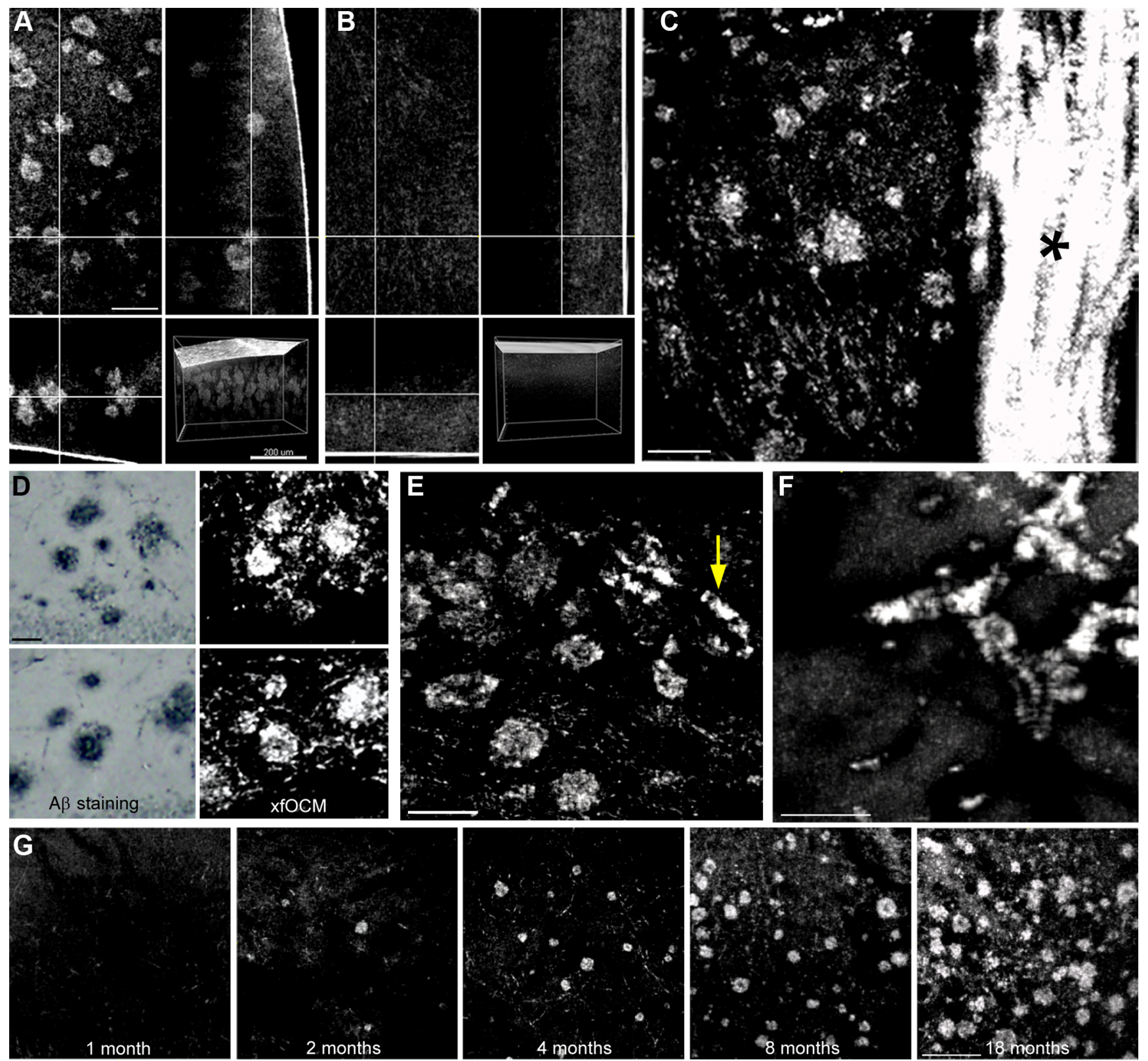

Figure 2. Ex vivo xfOCM imaging of $A \beta$ plaques in Alzheimeric brains. $A, A \beta$ plaques are imaged by xfOCM in the parietal cortex of an $A \beta$-depositing APPPS1 transgenic mouse brain, as shown in the top left. Scale bar, $100 \mu \mathrm{m}$. The same $A \beta$ plaque can also be visualized in the orthogonal $y$ and $z$ projections (top right and bottom left, respectively). Bottom right, Shows a $3 \mathrm{D}$ rendering of $A \beta$ plaques in a transgenic brain. $B$, xfOCM imaging in the parietal cortex of an aged-matched nontransgenic littermate is shown as control. $C$, Imaging on a coronal section from an $A \beta$-depositing APPPS1 mouse shows $A \beta$ plaques in the cortex (the figure is a maximum Z projection of $60 \mu \mathrm{m}$ ). Scale bar, $100 \mu \mathrm{m}$. Note the presence of the corpus callosum, as shown by the asterisk. $D$ Confirmation that imaged structures in $A \beta$-laden brains are amyloid plaques was obtained by comparison with immunohistochemical staining of the same brain regions using the anti-rabbit DW6 anti-A $\beta$ antibody. Left, Shows two examples of regions containing $A \beta$-stained plaques in the dentate gyrus. Scale bar, $50 \mu \mathrm{m}$. The same plaque morphologies were imaged by exvivo xfOCM (right). $\boldsymbol{E}$, Imaging APP23 brains reveal parenchymal A $\beta$ plaques and vascular A $\beta$ deposition (as shown by the arrow) whereas amyloid in APP Dutch mice $(\boldsymbol{F})$ is observed exclusively in the brain vasculature (figures are maximum Z projection of $50 \mu \mathrm{m}$ ). Scale bar, $100 \mu \mathrm{m}$. G, For the transversal study, predepositing (1-month-old) and depositing (2-, 4-, 8-, and 18-month-old) APPPS1 brains were imaged by xfOCM (figure are maximum Z projection of $50 \mu \mathrm{m}$ ). Scale bar, $100 \mu \mathrm{m}$.

tained from imaging APP23 brains revealed that both parenchymal and vascular amyloid are simultaneously reconstructed by xfOCM (Fig. 2E). Imaging APP Dutch brains unequivocally confirmed xfOCM imaging of cerebrovascular amyloid (Fig. $2 F$ ). Third, we have conducted a transversal xfOCM imaging study, using APPPS1 brains at different stages of amyloid pathology: predepositing (1 month old), young ( 2 and 4 months old), adult ( 8 months old), and aged (18 months old) A $\beta$-depositing mice (Figs. $2 G, 3 D$ ). $x y z$ stacks from the transversal study were processed per age group to obtain the $\mathrm{A} \beta$ plaque load and average plaque volume. Furthermore, all individual plaques detected in $x y z$ stacks from young, 2-month-old mice were categorized according to their radii (further details in methods). xfOCM permitted imaging of plaques as small as 3-6 $\mu \mathrm{m}$ in young depositing mice (Fig. $3 B$ ). The amyloid load and average plaque volume increased significantly with age $(p<0.001$ for both) (Fig. $3 B$ ), similar to these previously reported on postmortem $A \beta$ stained cortical sections of the same transgenic model (Radde et al., 2006).

We then proceeded to image cerebral amyloidosis in living adult $\mathrm{A} \beta$-depositing APPPS 1 mice using xfOCM through a small 
A

B
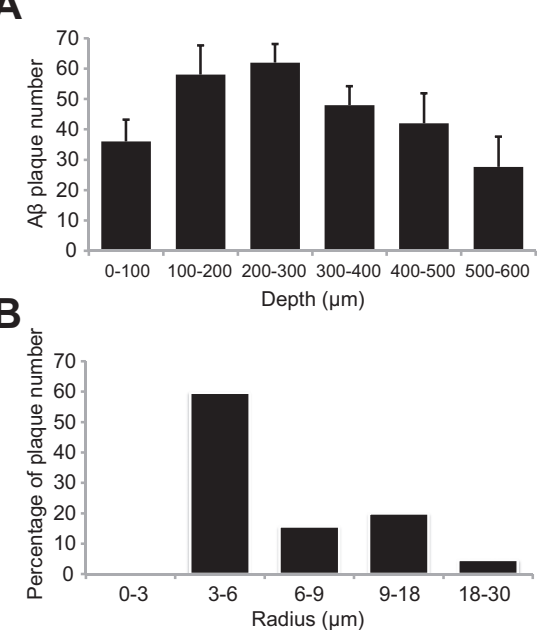

C

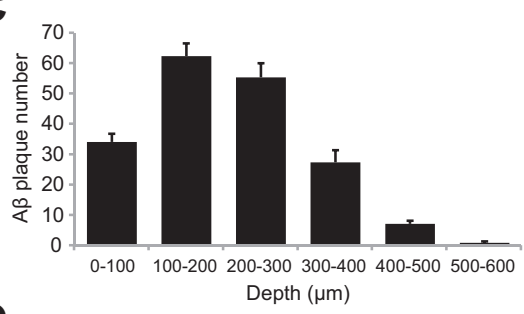

D
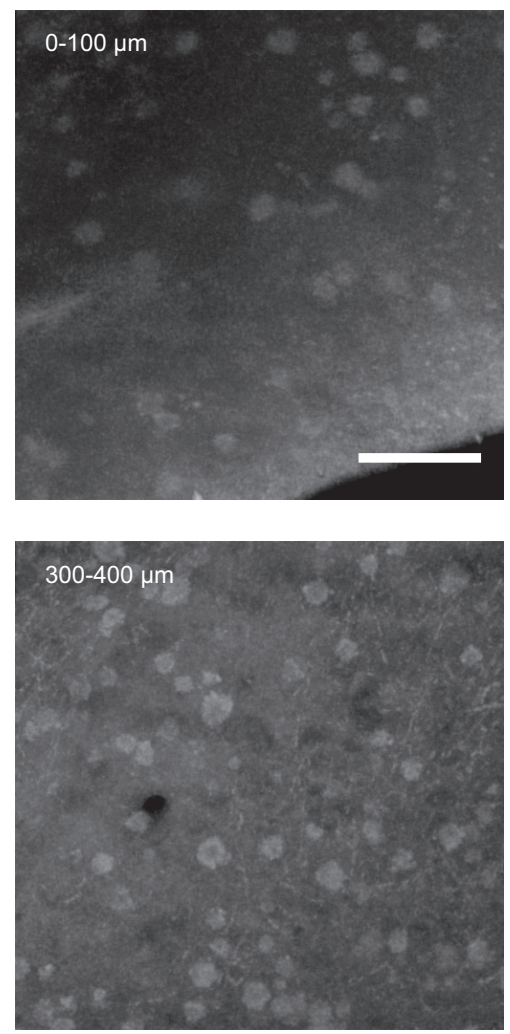
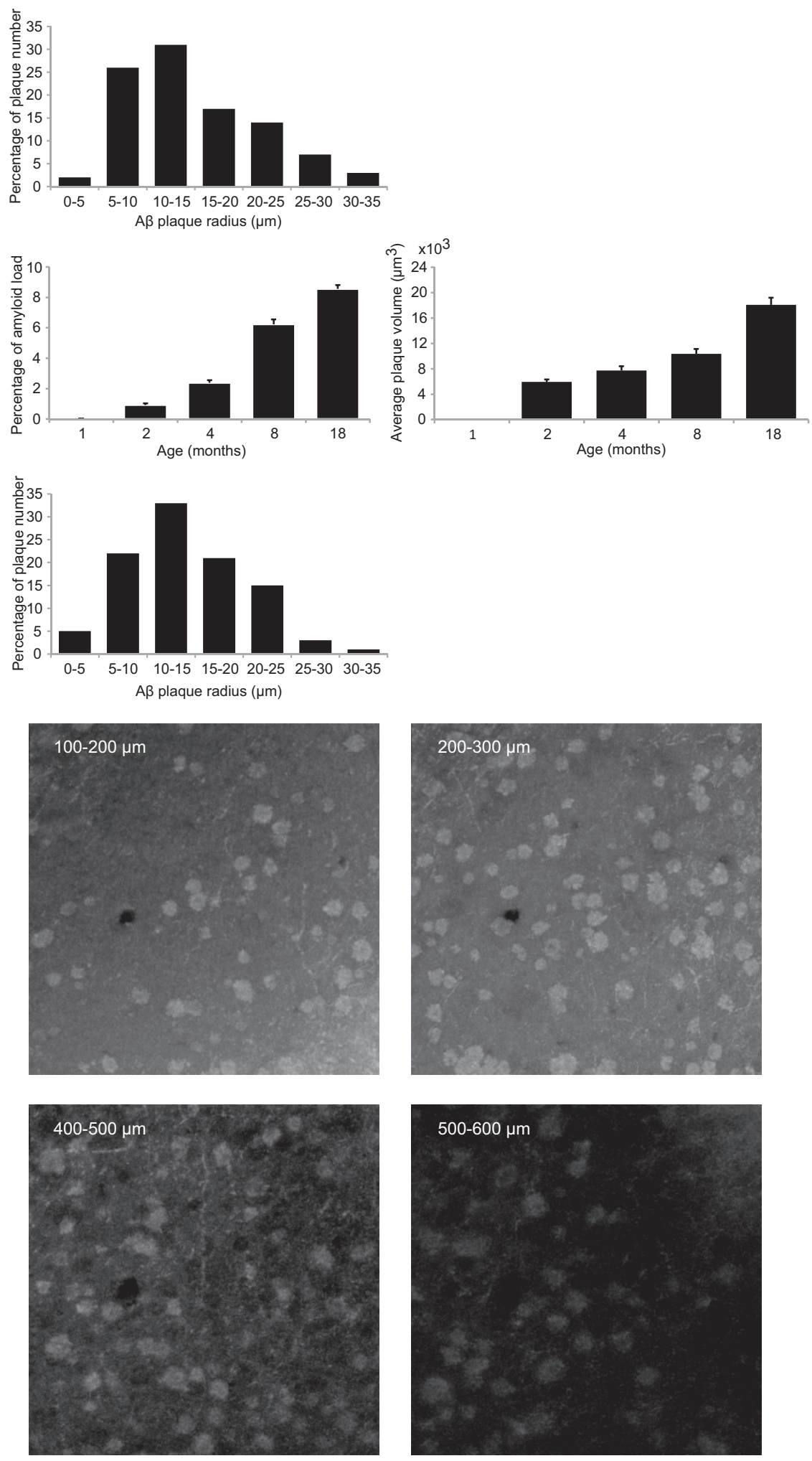

Figure 3. Characterization of $A \beta$ plaques imaged by xfOCM. $A, A \beta$ plaques distribution over $600 \mu \mathrm{m}$ below the surface of transgenic brains (left), and size distribution of all detected plaques detected over a depth range of $100-300 \mu \mathrm{m}$ below the cortical surface (right). $\boldsymbol{B}$, Individual plaques detected in xyz stacks from 2-month-old transgenic mice were categorized according to their radii (left). Quantification of A $\beta$ plaque load (middle) and average plaque volume (right) per age group of APPPS1 used in the transversal study. Statistical analysis revealed that the $A \beta$ plaque load and average volume significantly increase with age $(p<0.001$ for both). C, Distribution of $A \beta$ plaques over $600 \mu \mathrm{m}$ below the cortical surface of living APPPS1 mice (left), and size distribution of all detected plaques located over a depth range of $100-300 \mu \mathrm{m}$ below the brain surface (right). $\boldsymbol{D}$, Unprocessed maximum Z projections of $100 \mu \mathrm{m}$ at successive depths over $600 \mu \mathrm{m}$ below the cortical surface in an 8-month-old APPPS1 mouse, representative for the images used for quantification. Scale bar, $200 \mu \mathrm{m}$. 

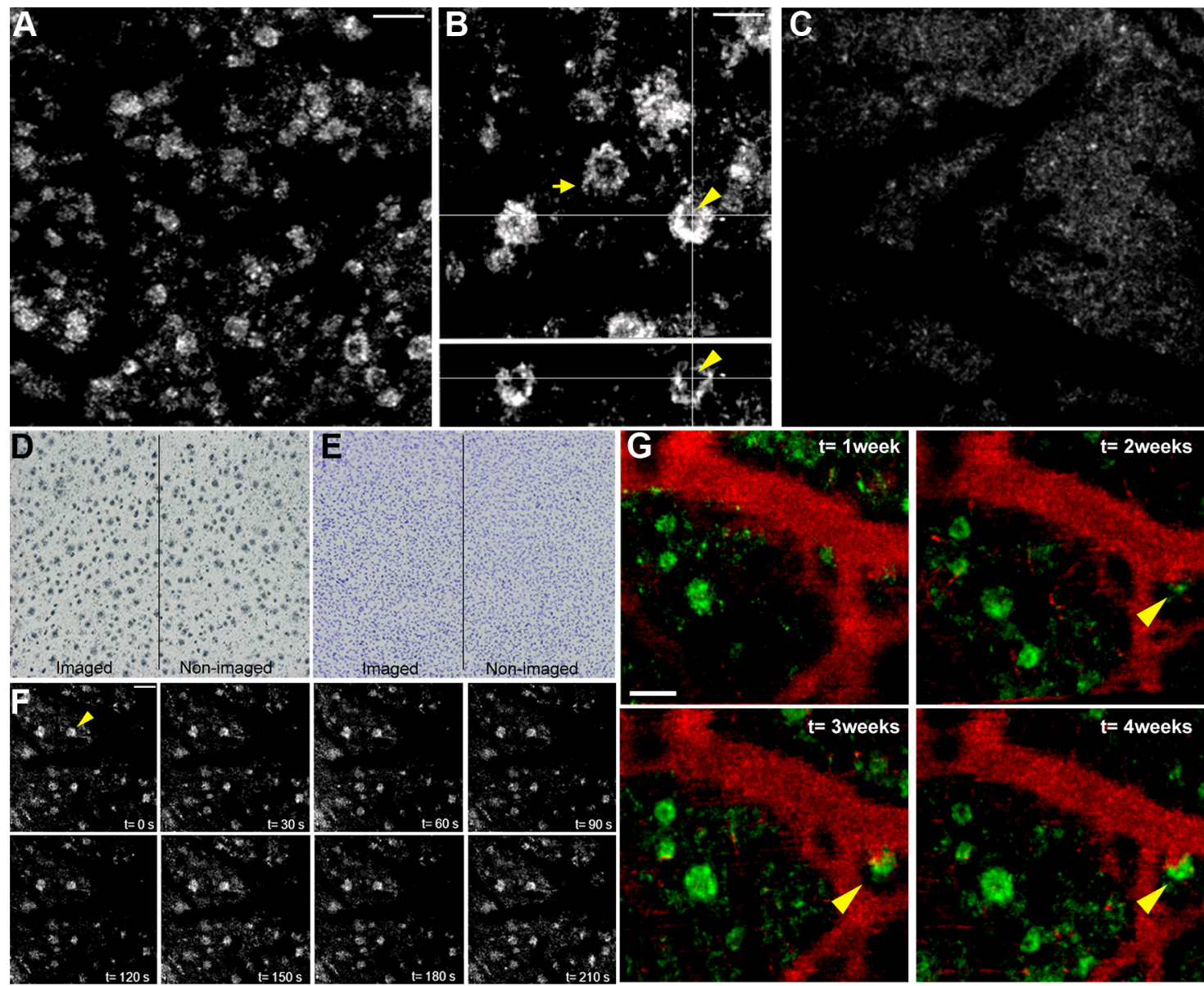

Figure 4. In vivo xfOCM imaging of cerebral $A \beta$ amyloidosis. $A$, Through a small craniotomy performed on the parietal cortex of an anesthetized APPPS1 mouse, numerous A $\beta$ deposits are imaged by xfOCM at a depth of $\sim 150 \mu \mathrm{m}$ below cortical surface (figure is a maximum Z projection of $50 \mu \mathrm{m}$ ). Scale bar, $75 \mu \mathrm{m}$. $\boldsymbol{B}$, Top panel, High-resolution scanning allows the detection of fine fibrils that emanate from $A \beta$ plaques (arrow symbol) and, in some cases, the dense plaque core itself (as indicated by an arrowhead) shows reduced light scattering (figure is a maximum $Z$ projection of $40 \mu \mathrm{m}$ ). Scale bar, $50 \mu \mathrm{m}$. Bottom, The same $A \beta$ plaque can further be visualized in the orthogonal y projection. $C$, No amyloid structures are reconstructed in the parietal cortex of a control, aged-matched, nontransgenic mouse monitored following the same procedures. $\boldsymbol{D}, \boldsymbol{E}$, Postmortem immunohistochemical $A \beta$ staining $(\boldsymbol{D})$ and cresyl violet histology $(\boldsymbol{E})$ of the imaged region by xfOCM versus surrounding, nonimaged area shows no evidence of a change in amyloid morphologies or a lesion as a result of imaging. The vertical line drawn in $\boldsymbol{D}$ and $\boldsymbol{E}$ represents the lateral edge of the cranial glass window. $\boldsymbol{F}$, Repeated imaging of the same amyloid-containing brain volume over a $240 \mathrm{~s}$ time frame (with eight sessions separated by $30 \mathrm{~s}$ ) reveals the overall short-term stability of $A \beta$ deposits (arrowhead shows one $A \beta$ plaque) (figure is a maximum $Z$ projection of $40 \mu \mathrm{m}$ ). Scale bar, $100 \mu \mathrm{m}$. G, Longitudinal imaging of the same plaque-containing volume in a young, 3-month-old APPPS1 mouse. Amyloid pathology was observed using structural xfOCM imaging (as shown in green), while xfOCM micro-angiography reveals the microvasculature (as shown in red), which was used for registering volumes over time (figures are maximum Z projections of $50 \mu \mathrm{m}$ ). Scale bar, $75 \mu \mathrm{m}$. Note the presence of a newly formed amyloid plaque in the imaged volume at $t=2$ weeks, that can be consistently followed thereafter, as shown by an arrowhead.

craniotomy performed over the parietal cortex of the animals. Image data collected from the parietal cortex consisted of $x y z$ stacks with $x y$ corresponding to $400^{2} \mu \mathrm{m}^{2}, 800^{2} \mu \mathrm{m}^{2}$, or $1000^{2}$ $\mu \mathrm{m}^{2}$, over a depth of $600 \mu \mathrm{m}$. A $\beta$ deposits could be readily detected up to $400-500 \mu \mathrm{m}$ below the cortical surface (Figs. $4 A, B$, $3 C$ ), without prior administration of an amyloid-binding dye or a radioactive tracer. The resolution of XfOCM images in vivo permitted the observation of the characteristic $A \beta$ fibrils extending from plaque cores (Fig. $4 B$ ). The amyloid structures imaged by $\mathrm{xfOCM}$ in APPPS1 mice were specific to $\mathrm{A} \beta$ plaques, as (1) no such objects could be observed in control, aged-matched, nontransgenic mice monitored following the same procedures (Fig. $4 C$ ) and (2) xfOCM images from young, predepositing APPPS1 mice were similar to those from nontransgenic mice (data not shown). Further assessment of cerebral tissue also showed no evidence of a change in amyloid morphologies or lesion caused by imaging, as evidenced by postmortem $\mathrm{A} \beta$ immunostaining or cresyl violet histology of the imaged regions versus surrounding, nonimaged areas (Fig. 4D,E, respectively). Overall, label-free
xfOCM allowed in vivo imaging of $\mathrm{A} \beta$ pathology with brain penetration similar to that obtained with multiphoton microscopy techniques using amyloid-binding dyes (Christie et al., 2001; Bacskai and Hyman, 2002; Klunk et al., 2002). To demonstrate that xfOCM provides stable imaging of cerebral $A \beta$ plaques over time, adult APPPS1 mice were imaged repeatedly at brief intervals (eight sessions with $30 \mathrm{~s}$ intersession). Additionally, we imaged $\mathrm{A} \beta$ plaques through a cranial glass window in the adult APPPS1 cortex over a longer time period (two sessions separated by $24 \mathrm{~h}$; data not shown). Last, we performed a one-month longitudinal study in young, 3-month-old APPPS1 mice (five imaging sessions each separated by 1 week). Analyses of $x y z t$ stacks revealed that plaque-containing volumes (with $x y z$ corresponding to $800^{2} \times 600 \mu \mathrm{m}^{3}$ ) could be consistently imaged in the living mouse cortex across all sessions in short-term (Fig. $4 F$ ) and long-term protocols (Fig. $4 G$ ). Consequently, xfOCM offers the unique opportunity to follow $\mathrm{A} \beta$ plaque growth and maintenance in the living brain in the absence of amyloid-binding dyes such as thioflavin and Congo red derivatives, which have been 

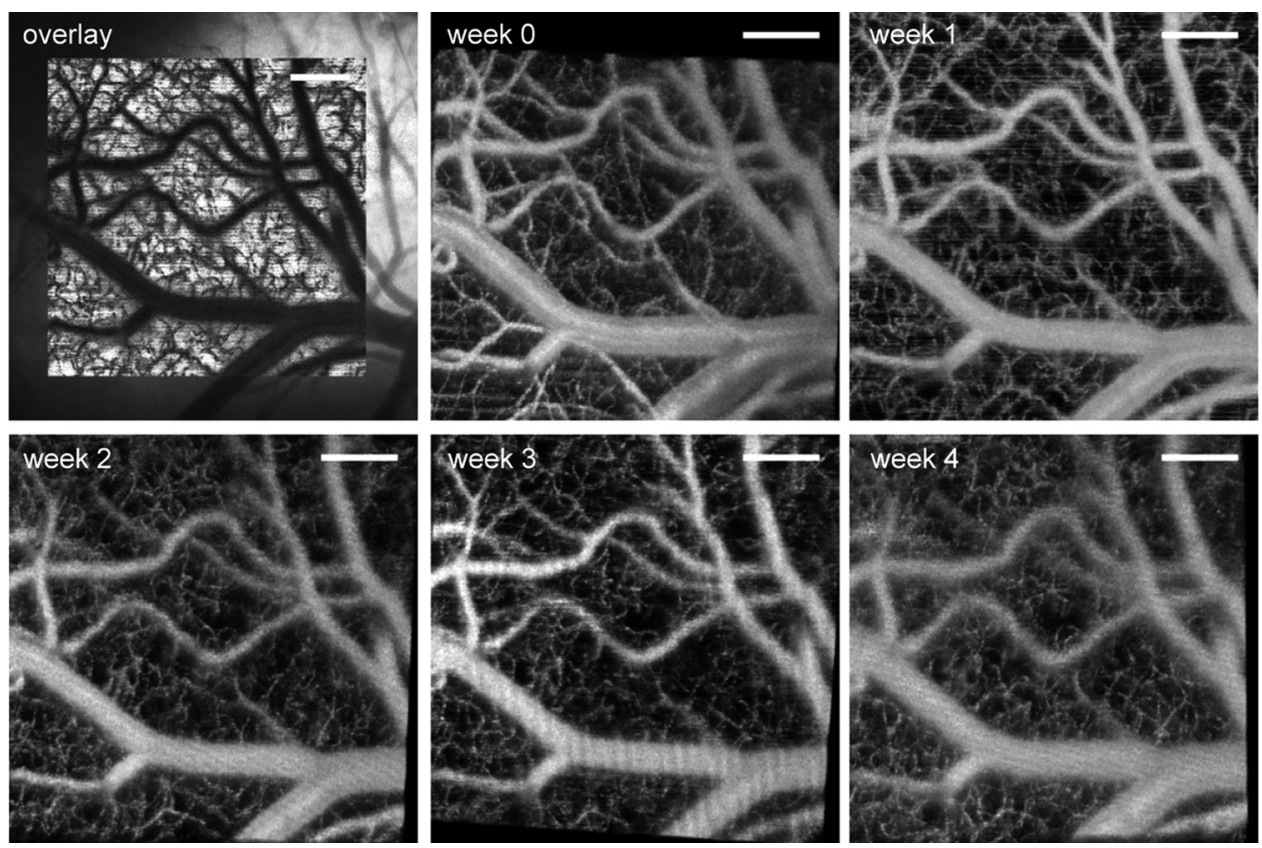

Figure 5. Label-free micro-angiography used for blood vessel registering. Top left, Live image taken through the microscope objective under white light illumination of large blood vessels under the mouse cranial glass window. Precise registration of the xfOCM image stacks over time was done on the 3D structure of the brain's vasculature (inset, top left, and other parts). The 3D structure of blood vessels was obtained by label-free optical micro-angiography of the same brain location (figures are maximum Z projections over the entire image stack). Scale bar, $150 \mu \mathrm{m}$.
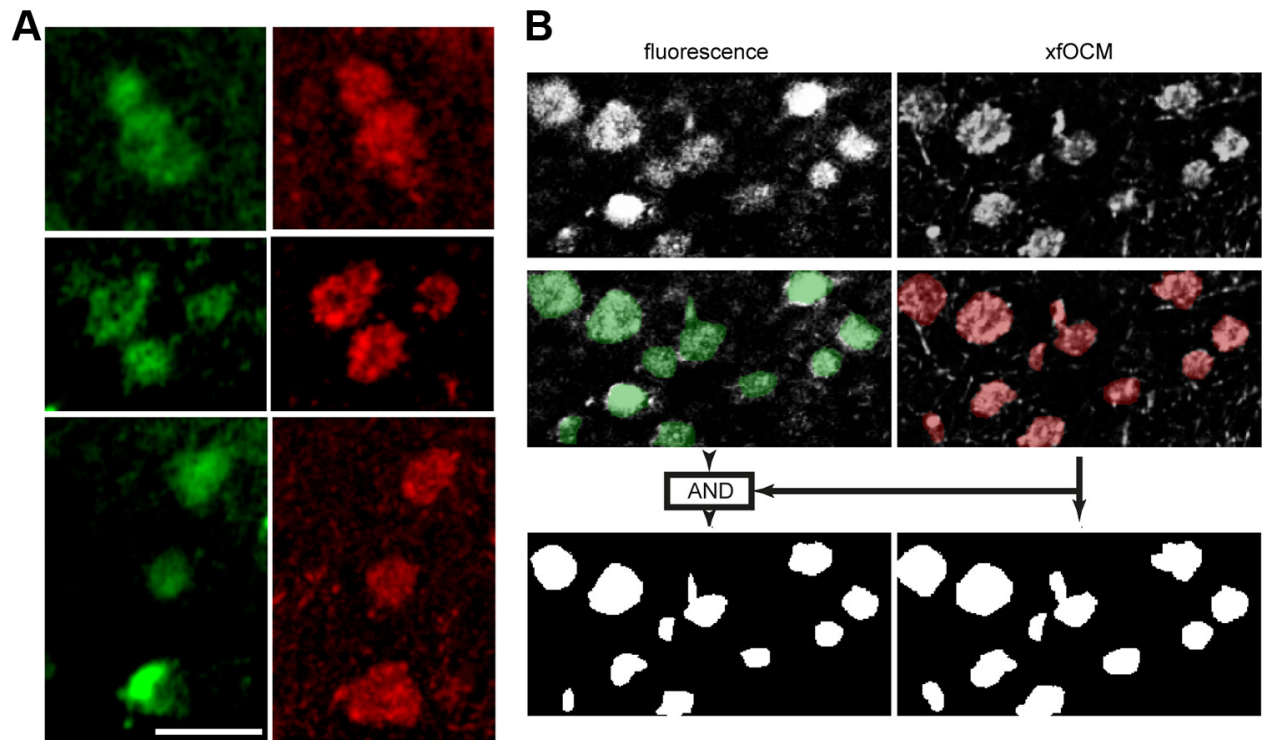

Figure 6. Comparison of $\mathrm{A} \beta$ plaque morphologies between xfOCM and fluorescence imaging. $A$, Imaged structures in $A \beta$-laden cortices were compared between fluorescence imaging (green) and xfOCM (red) of the same brain regions. Left, Shows three examples of regions containing $A \beta$-stained plaques in the neocortex. Scale bar, $75 \mu \mathrm{m}$. The same plaque morphologies were imaged simultaneously by ex vivo xfOCM (right). B, Quantification of the spatial overlap between plaque structures imaged using fluorescence (top left) and xfOCM (top right). Segmentation allowed identification of plaque areas in both modalities (middle and green and red overlays). The colocalization coefficient of xfOCM to fluorescence imaging was calculated as the ratio between the area jointly identified by the segmentations on both modalities as plaques (bottom left) to the total plaque area in the xfoCM image (bottom right).

reported to alter the formation of amyloid structures (Lee, 2002; Cohen et al., 2009). In the later imaging protocol spanning across several weeks, the evolution of existing amyloid plaques as well as the appearance of new deposits could be observed in label-free conditions (Fig. $4 G$ ), altogether very similar to that previously described using amyloid-binding agents in multiphoton studies (Bolmont et al., 2008; Hefendehl et al., 2011). Precise relocation of stacks over time was based on registration of 3D structure of the brain's vasculature obtained by label-free optical microangiography of the same brain area (Fig. 5). Micro-angiography images were acquired using a previously reported scan protocol (Srinivasan et al., 2010) and data stacks were processed using a phase variance algorithm (Vakoc et al., 2009) revealing only moving light scatterers such as red blood cells, thereby enabling the xfOCM instrument with a second contrast method. To demonstrate that morphologies of amyloid structures imaged by xfOCM are similar to that obtained by the traditional fluorescence method with an amyloid-binding dye, a confocal fluorescent imaging channel was added to the xfOCM setup, permitting simultaneous visualization of the same amyloid ensembles by the two modali- 
ties. This particular approach circumvents the imprecision and complications related to relocating individual amyloid plaques between two spatially separated imaging instruments. To ensure homogenous distribution ex vivo of the amyloid-binding agent in relatively thick tissue sections $(1000 \mu \mathrm{m})$, living APPPS1 mice were injected intraperitoneally with the dye methoxy-X04, a derivative of Congo red that crosses the blood-brain barrier, following a similar administration protocol as that used for in vivo multiphoton amyloid imaging (Bolmont et al., 2008; Hefendehl et al., 2011). After $24 \mathrm{~h}$, brains of injected $\mathrm{A} \beta$-laden animals were serial sectioned and subsequently observed without additional manipulation by simultaneous fluorescence microscopy and xfOCM. Fluorescence microscopic images of methoxy-X04 stained APPPS1 brain sections (Fig. $6 \mathrm{~A}$, left) revealed highly similar $\mathrm{A} \beta$ plaque morphologies to that imaged by xfOCM (Fig. $6 \mathrm{~A}$, right). Quantitative analysis of the spatial overlap between xfOCM imaging of amyloid plaques and images from amyloid binding methoxy-X04 dye was performed by first applying a segmentation on both images (Fig. $6 \mathrm{~B}$, top and middle). A colocalization coefficient analogous to Manders' overlap coefficient was then calculated from the fraction of xfOCM image area mutually identified by both modalities as plaques (Fig. $6 B$, bottom left) to the total area occupied by plaque in the xfOCM images (Fig. $6 B$, bottom right) (Manders et al., 1993). The coefficient as measured on the ensemble of images was calculated to be $83 \%$ (see Materials and Methods), indicating a high spatial overlap between $\mathrm{A} \beta$ plaques imaged by both modalities.

\section{Discussion}

Altogether, the data presented herein demonstrate that XfOCM is a powerful method to image cerebral $\mathrm{A} \beta$ amyloidosis ex vivo and in the living brain. Importantly, no prior $A \beta$ staining of brain samples was needed and tissue processing was not a prerequisite for effective ex vivo xfOCM imaging of cerebral $\mathrm{A} \beta$ amyloidosis. Furthermore, in vivo imaging of $\mathrm{A} \beta$ deposits required no administration of contrast agents, thereby precluding the appearance of unwanted variations in data collection due to interindividual and intraindividual variability in the uptake of amyloid dyes/radioactive tracers or in administration procedures. Overall, xfOCM offers advantages in terms of high-resolution and deep imaging in a minimally invasive way. The fast data acquisition makes xfOCM a highly practical technique for in vivo experiments, where measurement time is limited. Interestingly, the complementary capability of xfOCM to map vascular networks (Fig. 5) and image cerebral blood flow (Vakoc et al., 2009; Yaseen et al., 2011) will open new possibilities for label-free investigation of the vascular changes related to $\mathrm{A} \beta$ deposition in vivo. Thus, we anticipate that the use of xfOCM will help to improve the diagnosis and imaging of cerebral $A \beta$ amyloidosis in living animal models, with attendant translational applications to evaluate the efficacy of new $\mathrm{A} \beta$-targeting therapeutic strategies. In regard to the wide range of neurodegenerative disorders characterized by the abnormal aggregation of misfolded proteins other than $\mathrm{A} \beta$, forthcoming studies will also be aimed at implementing xfOCM for the diagnosis of neurofibrillary tau lesions and $\alpha$-synuclein-containing Lewy bodies.

\section{References}

Bacskai BJ, Hyman BT (2002) Alzheimer's disease: what multiphoton microscopy teaches us. Neuroscientist 8:386-390.

Bolmont T, Haiss F, Eicke D, Radde R, Mathis CA, Klunk WE, Kohsaka S, Jucker M, Calhoun ME (2008) Dynamics of the microglial/amyloid interaction indicate a role in plaque maintenance. J Neurosci 28:4283-4292.

Calhoun ME, Burgermeister P, Phinney AL, Stalder M, Tolnay M, Wieder- hold KH, Abramowski D, Sturchler-Pierrat C, Sommer B, Staufenbiel M, Jucker M (1999) Neuronal overexpression of mutant amyloid precursor protein results in prominent deposition of cerebrovascular amyloid. Proc Natl Acad Sci U S A 96:14088-14093.

Christie RH, Bacskai BJ, Zipfel WR, Williams RM, Kajdasz ST, Webb WW, Hyman BT (2001) Growth arrest of individual senile plaques in a model of Alzheimer's disease observed by in vivo multiphoton microscopy. J Neurosci 21:858-864.

Cohen AD, Ikonomovic MD, Abrahamson EE, Paljug WR, Dekosky ST, Lefterov IM, Koldamova RP, Shao L, Debnath ML, Mason NS, Mathis CA, Klunk WE (2009) Anti-amyloid effects of small molecule Abeta-binding agents in PS1/APP mice. Lett Drug Des Discov 6:437.

Fahrbach FO, Simon P, Rohrbach, A (2010) Microscopy with selfreconstructing beams. Nat Photonics 4:780-785.

Fercher AF, Drexler W, Hitzenberger CK, Lasser T (2003) Optical coherence tomography - principles and applications. Rep Prog Phys 66:239-303.

Fujimoto JG (2003) Optical coherence tomography for ultrahigh resolution in vivo imaging. Nat Biotechnol 21:1361-1367.

Hardy J, Selkoe DJ (2002) The amyloid hypothesis of Alzheimer's disease: progress and problems on the road to therapeutics. Science 297:353-356.

Hefendehl JK, Wegenast-Braun BM, Liebig C, Eicke D, Milford D, Calhoun ME, Kohsaka S, Eichner M, Jucker M (2011) Long-term in vivo imaging of beta-amyloid plaque appearance and growth in a mouse model of cerebral beta-amyloidosis. J Neurosci 31:624-629.

Herzig MC, Winkler DT, Burgermeister P, Pfeifer M, Kohler E, Schmidt SD, Danner S, Abramowski D, Stürchler-Pierrat C, Bürki K, van Duinen SG, Maat-Schieman ML, Staufenbiel M, Mathews PM, Jucker M (2004) Abeta is targeted to the vasculature in a mouse model of hereditary cerebral hemorrhage with amyloidosis. Nat Neurosci 7:954-960.

Izatt JA, Hee MR, Owen GM, Swanson EA, Fujimoto JG (1994) Optical coherence microscopy in scattering media. Opt Lett 19:590-592.

Klunk WE, Bacskai BJ, Mathis CA, Kajdasz ST, McLellan ME, Frosch MP, Debnath ML, Holt DP, Wang Y, Hyman BT (2002) Imaging Abeta plaques in living transgenic mice with multiphoton microscopy and methoxy-X04, a systemically administered Congo red derivative. J Neuropathol Exp Neurol 61:797-805.

Lee VM (2002) Amyloid binding ligands as Alzheimer's disease therapies. Neurobiol Aging 23:1039-1042.

Leitgeb RA, Villiger M, Bachmann AH, Steinmann L, Lasser T (2006) Extended focus depth for Fourier domain optical coherence microscopy. Opt Lett 31:2450-2452.

Leitgeb R, Hitzenberger C, Fercher A (2003) Performance of fourier domain vs. time domain optical coherence tomography. Opt Express 11:889-894.

Manders EMM, Verbeek FJ, Aten JA (1993) Measurement of colocalization of objects in dual-color confocal images. J Microsc 169:375-382.

Radde R, Bolmont T, Kaeser SA, Coomaraswamy J, Lindau D, Stoltze L, Calhoun ME, Jäggi F, Wolburg H, Gengler S, Haass C, Ghetti B, Czech C, Hölscher C, Mathews PM, Jucker M (2006) Abeta42-driven cerebral amyloidosis in transgenic mice reveals early and robust pathology. EMBO Rep 7:940-946.

Srinivasan VJ, Jiang JY, Yaseen MA, Radhakrishnan H, Wu W, Barry S, Cable AE, Boas DA (2010) Rapid volumetric angiography of cortical microvasculature with optical coherence tomography. Opt Lett 35:43-45.

Sturchler-Pierrat C, Abramowski D, Duke M, Wiederhold KH, Mistl C, Rothacher S, Ledermann B, Bürki K, Frey P, Paganetti PA, Waridel C, Calhoun ME, Jucker M, Probst A, Staufenbiel M, Sommer B (1997) Two amyloid precursor protein transgenic mouse models with Alzheimer disease-like pathology. Proc Natl Acad Sci U S A 94:13287-13292.

Vakoc BJ, Lanning RM, Tyrrell JA, Padera TP, Bartlett LA, Stylianopoulos T, Munn LL, Tearney GJ, Fukumura D, Jain RK, Bouma BE (2009) Threedimensional microscopy of the tumor microenvironment in vivo using optical frequency domain imaging. Nat Med 15:1219-1223.

Villiger M, Goulley J, Friedrich M, Grapin-Botton A, Meda P, Lasser T, Leitgeb RA (2009) In vivo imaging of murine endocrine islets of Langerhans with extended-focus optical coherence microscopy. Diabetologia 52:1599-1607.

Yaseen MA, Srinivasan VJ, Sakadžiæ S, Radhakrishnan H, Gorczynska I, Wu W, Fujimoto JG, Boas DA (2011) Microvascular oxygen tension and flow measurements in rodent cerebral cortex during baseline conditions and functional activation. J Cereb Blood Flow Metab 31:1051-1063.

Zhang Y, Brady M, Smith S (2001) Segmentation of brain MR images through a hidden Markov random field model and the expectationmaximization algorithm. IEEE Trans Med Imaging 20:45-57. 